\title{
Dental stem cells: a future asset of ocular cell therapy
}

\author{
GARY HIN-FAI YAM ${ }^{1,2}$, GARY SWEE-LIM PEH ${ }^{1,2}$, SHWETA SINGHAL ${ }^{3}$, BEE-TIN GOH ${ }^{4}$, \\ JODHBIR S. MEHTA ${ }^{1,2,3 *}$ \\ ${ }^{1}$ Tissue Engineering and Stem Cell Group, Singapore Eye Research Institute, Singapore, ${ }^{2}$ Eye-ACP, Duke-NUS \\ Graduate Medical School, Singapore, ${ }^{3}$ Singapore National Eye Centre, Singapore, and ${ }^{4}$ Department of Oral and \\ Maxillofacial Surgery, National Dental Centre, Singapore
}

\begin{abstract}
Regenerative medicine using patient's own stem cells (SCs) to repair dysfunctional tissues is an attractive approach to complement surgical and pharmacological treatments for aging and degenerative disorders. Recently, dental SCs have drawn much attention owing to their accessibility, plasticity and applicability for regenerative use not only for dental, but also other body tissues. In ophthalmology, there has been increasing interest to differentiate dental pulp SC and periodontal ligament SC (PDLSC) towards ocular lineage. Both can commit to retinal fate expressing eye field transcription factors and generate rhodopsin-positive photoreceptor-like cells. This proposes a novel therapeutic alternative for retinal degeneration diseases. Moreover, as PDLSC shares similar cranial neural crest origin and proteoglycan secretion with corneal stromal keratoctyes and corneal endothelial cells, this offers the possibility of differentiating PDLSC to these corneal cell types. The advance could lead to a shift in the medical management of corneal opacities and endothelial disorders from highly invasive corneal transplantation using limited donor tissue to cell therapy utilizing autologous cells. This article provides an overview of dental SC research and the perspective of utilizing dental SCs for ocular regenerative medicine.
\end{abstract}

\section{Introduction}

Stem cell (SC) research offers new strategies to treat degenerative diseases, for which few effective treatments currently exist. Many studies have aimed at repairing aging and diseased organs by the replacement of damaged cells with healthy functional cells that are cultivated or produced in the laboratory or within organs (e.g. liver, mammary glands and prostate) regenerated from SCs, either from autologous origin or allogeneic donor tissue (Refs 1, 2, 3, 4). The implantation of new cells could also trigger paracrine signalling (via diffusible factors and/or niche matrix interactions) to mediate endogenous growth and the regeneration of undamaged resident cells in target tissue (Refs 5,6). This concept of 'regenerative medicine' offers novel treatment tools for degenerative diseases.

The population of pluripotent embryonic SCs (ESCs) is an extremely attractive cell source for lineage cell differentiation and tissue regeneration. However, the risk of immunogenicity and rejection, tumour formation and ethical considerations have restricted their use to mainly in vitro experimental studies and their therapeutic potential remains to be determined. To date, the first trials of human ESCs have begun and completed result will only be available in coming years. In 2010, a phase 1 clinical trial of transplanting human ESC-derived oligodendrocyte progenitors GRNOPC1 was performed on four patients with spinal cord injuries. The participants received a single injection of GRNOPC1 ( $\sim 2$ million cells) and they experienced neither serious adverse events nor any change to spinal cord neurological condition (Ref. 7). The treatment efficacy will only be concluded after a long-term follow-up of these patients (Ref. 8). In a phase $1 / 2$ study transplanting human ESC-derived retinal pigment epithelial (RPE) cells to the subretinal space in a total of 18 patients with advanced dry type of age-related macular degeneration (AMD) or Stargardt's disease, there was no adverse cell proliferation and rejection and patients had improved visual acuity (Ref. 9). Though these results are encouraging, long-term safety and graft survival have to be fully elucidated in order to validate ESC therapy as a safe treatment for degenerative diseases. Today ESCs are also headed for trials in diabetes and heart failure. The advances of induced pluripotent stem cells (iPSCs) offer the possibility of obtaining abundant amounts of undifferentiated ESC-like cells from allogenic or autologous somatic cells for tissue and drug development purposes (Ref. 10). However, there are different methodologies to generate iPSCs with varied efficiencies, hence creating problems in reproducibility and SC maintenance (Refs 12, 13). When using adenoand/or retro-viruses to introduce core pluripotent transcription factors to somatic cells, transgene sequences 
could be encrypted in the host genome, increasing the likelihood of tumorigenesis and hence hindering future translational applications. Zero-footprint methods such as reprogramming by episomal factors, mini-circles, piggyBac, direct microRNA transfection and mRNA and protein over-expression of reprogramming factors with small molecule induction can substantially reduce the chance of tumorigenicity caused by random chromosomal insertion of exogenous genes. However, these different methodologies will generate iPSCs with varied efficiencies, hence creating problems in the reproducibility of pluripotent cell generation, maintenance and differentiation (Refs 11, 12, 13). Forced expression of reprogramming factors cannot be controlled fully, leading to unpredictable effects. The reprogramming efficiency must also be improved through identification and manipulation of a myraid of molecular pathways that are implicated in somatic cell reprogramming. Another major concern is that iPSCs retain the intrinsic memory of the original tissue and this may cause the cells to work incorrectly as pluripotent cells and affect the eventual functionality of the desired differentiated cell type (Refs 14, 15). They also tend to age prematurely and have a higher rate of apoptosis, hence a clear understanding of iPSC fate regulation and improvement in long-term survival is required (Ref. 16).

In contrast, multipotential somatic SCs or tissue-specific adult SCs are present in virtually all adult tissues. They are present in small numbers but divide in response to injury to generate cells for tissue maintenance and repair. Adult tissues that are known to contain SCs include skin and hair follicles (epidermal), bone marrow and peripheral blood (mesenchymal/hematopoietic), liver and skeletal muscle (mesenchymal) as well as brain (neural) (Ref. 17). Since they can differentiate and replenish specialized cells within the organ they reside, the concept of 'adult SC therapy' (ASCT) is becoming a treatment strategy for degenerative diseases. ASCT involves ex vivo manipulations (including cell isolation, enrichment and identification) and growth of adult SCs, which will be used to replace dysfunctional cells in the diseased organs (Ref. 18). Since the late 1960s, bone marrow SC transplantation has been performed to treat blood disorders, such as leukaemia and lymphoma (Ref. 19). Epidermal SCgenerated epidermis has served as skin grafts for patients with large-scale third degree burns however the new skin is devoid of hair follicles, sweat and sebaceous glands (http://www.EuroStemCell.org). Mesenchymal SC (MSC) therapy (from bone marrow and adipose tissue) has shown promising outcomes when used for tissue repair in cases of liver cirrhosis and failure, musculoskeletal defects, periodontal tissue defects, diabetic critical limb ischaemia, bone damage caused by osteonecrosis and burn-induced skin defects (Refs 20,21). In addition, MSC transplant can alleviate immune disorders, reverse graft-versushost disease, suppress inflammation and improve the treatment outcome of multiple system atrophy, multiple sclerosis, amyotrophic lateral sclerosis and stroke (Refs 21, 22). Transplantation of bone marrow MSC (BM-MSC)-derived dopaminergic cells to brain striatum of Parkinsonian monkeys showed the expression of dopamine transporter in the engrafted tissue and improved the recipients' motor behaviour (Ref. 23). The 'first-in-man' clinical trial of autologous BMMSC injection to 25 patients with progressive supranuclear palsy has proven this approach to be safe for humans, however therapeutic effectiveness was not achieved (Ref. 24). SC therapy for the treatment of diabetes mellitus is an alternative approach to islet transplantation, which is restricted by limited donor and graft rejection (Ref. 25). The protocol of differentiating SCs into insulin-secreting cells and its translational effect has been established for pluripotent cells and for adult MSC (Refs 26, 27, 28, 29). Rat BM-MSC and BM stromal cells were capable to trans-differentiate into insulin-positive cells with the addition of pancreatic extract (Ref. 30). These islet-like cells could lower the circulating glucose levels after sub-capsular renal transplantation (Ref. 31).

The goal of ASCT is to introduce healthy cells to reside and differentiate to form functional cells in the target tissue to repair the disease state. The mechanisms involved in these processes are not fully elucidated, despite evidence suggesting that some transplanted cells were able to survive and integrate in the host tissue. These resident cells also exert paracrine effects through releasing cytokines and growth factors, which stimulate the growth of resident and functionally competent cells (Refs 5, 32).

\section{Eye disorders: an opportunity of SC-based therapy}

The human eye is an immune-privileged organ, meaning that transplanted cells are not as likely to be rejected as foreign materials compared with transplants elsewhere. In a phase I/II trial, patients with AMD and Stargardt's macular dystrophy received SC therapy by transplanting human ESC-derived RPE cells. Besides the primary goal of safety assessment, the treatment delayed disease progression and improved visual acuity (Ref. 9). Further assessment will underpin if the cells are well received by the body's immune system or become overactive and grow into tumours. Long-term stability, survival and functionality of transplanted cells generated by chemical induction of human ESCs are also critical concerns before concluding the success of ESC therapy.

'Corneal opacities' are characterised by scarring and clouding of the cornea, and they reduce vision (Ref. 33). Trauma, infection, immunological disorders and, inherited diseases and degeneration (such as corneal dystrophies and keratoconus) and/or induced injuries (e.g. surgical trauma) can lead to corneal opacities and a reduction in visual acuity, and even corneal blindness. Infection-induced opacities are manifested 
acutely and most scarring can be reduced after appropriate therapy. However, residual scars that might be left in the central/para-central cornea will cause a visual deficit. Non-infectious and non-inflammatory corneal opacities usually develop gradually (depositional or scarring disorders) and remain permanently. Over 10 million people worldwide are affected by corneal opacities, with a higher prevalence among Asians and Africans than Caucasians. However, a substantial number of patients with bilaterally corneal blindness $(\sim 4.9$ million worldwide estimated by WHO 2010 global blindness data and WHO 2002 sub-region causes) could potentially have their eyesight restored if the corneal opacification was removed (Refs 34, 35).

Corneal opacities are treated either medically or surgically. The choice of therapy depends on the severity, nature and aetiology of the scarring. In cases of infectious keratitis, when medical treatment is ineffective or if there is opacity progression, surgery will be considered. Corneal transplantation, either penetrating keratoplasty or lamellar procedures (anterior lamellar keratoplasty) may have to be performed either as acute therapeutic procedure or as a tectonic procedure (Refs $36,37,38)$. Even in cases following the resolution of infection, surgery may be needed to provide visual rehabilitation to remove corneal scarring (Ref. 39). Despite surgical advancement in the past decade, the surgical outcome is highly constrained by worldwide shortage of donor material, long-term graft survival, allograft rejection and high surgical and rehabilitation cost (Ref. 38). Hence, there is a need to explore alternative strategies, which are simple and less invasive to permanently eliminate or prevent stromal scarring at a reasonable cost. In order to achieve this purpose, a cell therapy involving an autologous SC source is an attractive option.

\section{Trans-differentiation potential of adult SCs into other lineage cells}

Adult SCs have been shown to have reprogramming potential and can trans-differentiate into different cell types other than those in which they reside. Unlike iPSCs, the conversion of adult SCs is shown to be more direct, efficient and more importantly, it bypasses the pluripotent cell state, which often elicits safety concerns and risk of tumorigenesis. An example is the conversion of adult mouse pancreatic exocrine cells to insulin-producing $\beta$-cells through the expression of transcription factors ( $P d x l, N g n 3$ and Mafa) in vivo (Refs 40, 41). The resultant cell morphology, cell marker expression and insulin secretion profile were similar to the native islet $\beta$-cells. However, subsequent transplantation studies did not show any integration of engineered cells with the existing islets. Functional cardiomyocytes were generated by retroviral-mediated expression of Gata4, Mef2c and Tbx5 in mouse cardiac fibroblasts (Ref. 42) and mature neurons have been formed after the forced expression of Asc11, Brn2a and Myt11 in mouse astrocytes (Ref. 43).
These studies highlight the trans-differentiation capacity of a variety of adult somatic cell types, and that transcription factors play a key role in their differentiation towards the desired target cell types. However, engraftment in the appropriate 'niche' also greatly influences cell growth and ultimate cell fate determination. The differentiation of BM-MSC towards cardiomyocyte fate was achieved by its engraftment in an appropriate 'niche' and the presence of trophic factors, rather than by the intrinsic cell program (Ref. 44). Though the exact mechanism remains undetermined, the provision of a suitable microenvironment and enhancement of functional activity of the specific niche (through short- and long-ranging signal mediators) may suffice to drive the differentiation of somatic SCs towards the desired cellular phenotype in their target tissue.

\section{Dental SCs}

There are two major types of dental SCs: dental MSClike cells and dental epithelial SCs. The latter is found in the continuously growing incisors of rodent and in molars of some mammalian species (Refs 45, 46). In 2000, Gronthos et al. first isolated MSC-like cells from human postnatal dental pulp [dental pulp SCs (DPSCs)] (Ref. 47). Subsequently, different types of MSC-like cells were identified from developing tooth [dental follicle SCs (DFSC)] (Ref. 48), postnatal tooth with periodontal ligament [periodontal ligament SCs (PDLSCs)] (Ref. 49) and apical papilla (stem cells from apical papilla) (Ref. 50) as well as the pulp cavity of exfoliated deciduous teeth (SCs from human exfoliated deciduous teeth) (Ref. 51) (Fig. 1). There are many reports on the dental SC characterisation and differentiation potential and information are summarised in Table 1 (Refs 52, 53, 54, 55). Owing to the predominant MSC features, all these dental SCs can differentiate into adipogenic, chondrogenic and osteo/odontogenic lineages, similar to BM-MSC. As a component of tooth development, dental SCs appear to be more committed to odontogenic fate, rather than osteogenic (Ref. 52). Since they are derived from post-migratory cranial neural crest cells, they have stronger neurogenic potential than BMMSC and may contain properties analogous to neural crest progenitor cells even at postnatal stages (Ref. 54). During development, cranial neural crest cells originated from the roof plate of neural tube (cranial region) migrate dorsolaterally to derive craniofacial mesenchyme, which further differentiates into cranial ganglia, craniofacial cartilage and bones. When they reach the pharyngeal pouches, they become cells in thymus, middle ear ossicles, jaw, tooth primordia [periodontal ligament (PDL) and dental pulp]. They also migrate to the ocular region and become periocular mesenchyme giving rise to corneal stromal keratocytes (CSKs) and corneal endothelial cells (CECs) (Refs 56, 57, 58). 


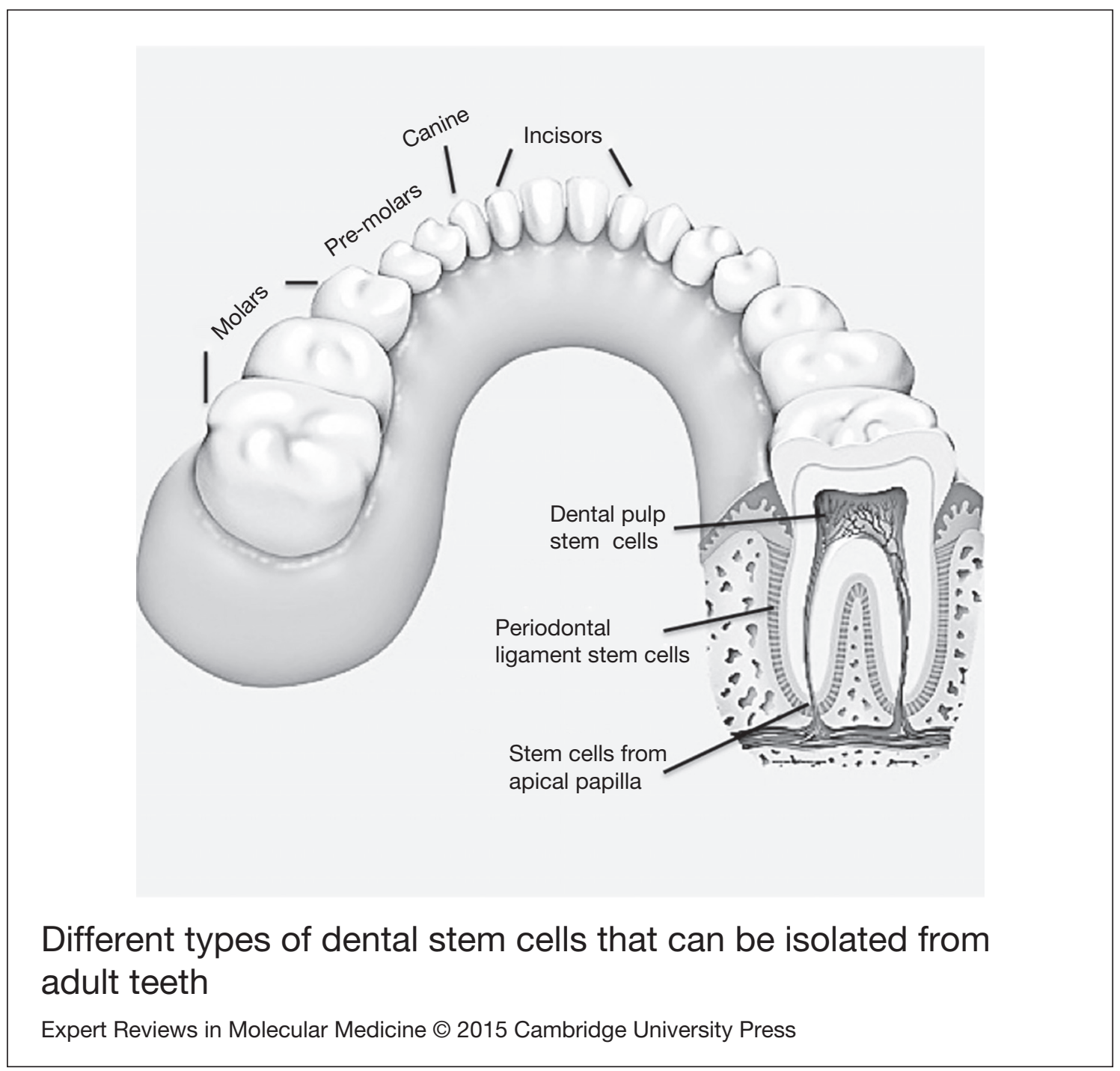

FIGURE 1

Different types of dental stem cells that can be isolated from adult teeth.

\section{Dental pulp SCs}

Dental pulp is the non-mineralized tissue at the centre of tooth. The pulp cavity is occupied by soft connective, vascular, lymphatic and nervous components that nourish and maintain the tooth. After physical removal of tooth crown and root, the pulp tissue is dissociated to single cells with a digestion mixture of collagenase, dispase and trypsin. Under serum culture, colony-forming cells are generated at a higher frequency from dental pulp (22-70 colonies/ $10^{4}$ cells plated) than from bone marrow stromal cells (Refs 59, 60) and they express various markers as depicted in Table 1. Under the influence of growth factors, such as leukaemia inhibitory factor, epidermal growth factor and platelet-derived growth factor, they undergo clonal proliferation forming colonies of small round rapidly dividing cells that constitutively express stage-specific embryonic antigen-4 (SSEA4), $O C T 3 / 4$ and NANOG while maintaining genomic stability, akin to pluripotent-like SCs (Ref. 61). Hilkens et al. found no significant difference in marker expression and differentiation potentials when DPSCs that were isolated by enzymatic digestion compared with explant growth from intact tissue (Ref. 62). Owing to the small volume size of dental pulp, a small number of non-vascular, lymphatic and neuronal cells could be isolated. Lizier et al. reported that DPSCs could be cultivated for 6 months without changes in morphology or SC marker expression, suggesting a prospect that these cells can be propagated to larger numbers for potential regenerative applications (Ref. 63). Viable DPSCs with appropriate marker expression could also be isolated from cryopreserved teeth under controlled cooling condition without cryoprotectants (such as dimethyl sulfoxide), demonstrating the applicability of tooth banking (more discussion in the following section) (Refs 64, 65).

DPSCs are capable of differentiating into a wide variety of tissue types, including osteocytes, myocytes, chondrocytes and adipocytes (Table 1) (Refs 66, 67, $68,69)$. They also share common expression markers (ABCG2, integrin $\beta 1$, vimentin, p63, and connexin 43) 
TABLE 1.

DENTAL STEM CELLS: IDENTIFICATION AND DIFFERENTIATION POTENTIAL

\begin{tabular}{|c|c|c|c|c|c|}
\hline Stem cell type & DFSC & SHED & DPSC & PDLSC & SCAP \\
\hline Dental tissue & Follicle & Pulp & Pulp & PDL & Apical papilla \\
\hline Tooth type & Dental germ & Primary & Permanent & Permanent & Permanent \\
\hline \multirow[t]{4}{*}{ Stemness markers } & ESC: nil & ESC: OCT4, Nanog & ESC: OCT4, Nanog & ESC: OCT4 & ESC: OCT4 \\
\hline & $\begin{array}{l}\text { MSC: CD44, 73, 90, 105, } \\
\text { 146, 166; STRO-1 }\end{array}$ & $\begin{array}{l}\text { MSC: CD44, 73, 90, 105, } \\
\text { 146, 166, STRO-1 }\end{array}$ & $\begin{array}{l}\text { MSC: CD44, 73, 90, 105, } \\
\text { 146, 166, STRO-1 }\end{array}$ & $\begin{array}{l}\text { MSC: CD44, 73, 90, 105, } \\
\text { 146, 166, STRO-1 }\end{array}$ & $\begin{array}{l}\text { MSC: CD44, 73, 90, 105, 106, } \\
\quad \text { 146, 166, STRO-1 }\end{array}$ \\
\hline & $\begin{array}{l}\text { SC: SSEA4, CD9, 13, 24, } \\
\text { 29, nestin, Notch1 }\end{array}$ & SC: SSEA4, nestin, Notch1 & $\begin{array}{l}\text { SC: SSEA4, CD9, 13, 29, } \\
\text { Notch1 }\end{array}$ & $\begin{array}{l}\text { SC: CD9, 13, 29, connexin } 43 \\
\text { NC: Slug, snail, Sox } 10 \text {, nestin }\end{array}$ & $\begin{array}{l}\text { SC: SSEA4, CD9, 13, 24, } 29 \text {, } \\
\text { nestin }\end{array}$ \\
\hline & & & $\begin{array}{l}\text { NSC: nestin, NeuN, } \beta 3- \\
\text { tubulin }\end{array}$ & NSC: NeuN, $\beta 3$-tubulin & $\begin{array}{l}\text { NC: Slug, snail, Sox } 10 \text {, nestin } \\
\text { HSC: CD } 80,86\end{array}$ \\
\hline \multicolumn{6}{|c|}{$\begin{array}{l}\text { Differentiation potential } \\
\text { (experimental) } \\
\text { (A) Dental }\end{array}$} \\
\hline Odontogenic & + & + & + & + & + \\
\hline Dentinogenic & ND & + & + & ND & ND \\
\hline Cementogenic & + & ND & ND & + & + \\
\hline \multicolumn{6}{|l|}{ (B) Non-dental } \\
\hline Osteogenic & + & + & + & + & + \\
\hline Chondrogenic & + & + & + & + & + \\
\hline Adipogenic & + & + & + & + & + \\
\hline Myogenic & + & + & + & + & ND \\
\hline Neurogenic & + & + & + & + & + \\
\hline (C) Ocular & ND & ND & $+(\mathrm{CSK})$ & $+(\mathrm{PR})$ & ND \\
\hline
\end{tabular}

CSK, corneal stromal keratocytes; DFSC, dental follicle stem cells; DPSC, dental pulp stem cells; ESC, embryonic stem cells; HSC, hematopoietic stem cells; MSC, mesenchymal stem cells; NC, neural crest; ND, no detection; NSC, neural stem cells; OCT4, octamer 4; SHED, stem cells from exfoliated deciduous teeth; PDLSC, periodontal ligament stem cell; PR, photoreceptors; SCAP, stem cells from apical papilla; SCs, stem cells (general); SSEA4, stage-specific embryonic antigen-4. 
with limbal SCs in the eye (Ref. 70). Transplantation of a tissue engineered cell sheet composed of undifferentiated immature human DPSCs to a rabbit alkali burn model of limbal SC deficiency (LSCD) yielded a reconstructed corneal epithelium expressing cytokeratin $3 / 12$ and 18 , reduced neovascularization and clear cornea. These results suggested that DPSC could be an alternative cell source for the corneal reconstruction of LSCD (Ref. 71).

\section{Neuronal differentiation potential of DPSC}

Given their neural crest origin, DPSCs have significant neuro-regenerative potential (Refs 72, 73, 74). They constitutively express early neuronal markers (such as nestin) and retain neuro-ectodermal features. When placed under neural induction condition with chemicals, such as retinoic acid (RA), neurotrophin-3, nerve growth factor (NGF), cyclic adenosine monophosphate (cAMP) and basic fibroblast growth factor (bFGF), DPSCs differentiated to express mature neuronal markers ( $\beta$-tubulin III and neurofilament-M) and activated voltage-gated sodium pump channels (Refs 75, 76). After transplantation into the brain of newborn rats, DPSCs exhibited homing to the sites of cortical lesion indicating their potential in rescuing or replacing damaged neurons (Ref. 76). They are known to exert paracrine neuro-tropism through their vast expression of growth factors, including glial-derived neurotrophic factor, ciliary neurotrophic factor and brain-derived neurotrophic factor and NGF (Refs 77, 78). When transplanted into models of rat spinal cord injury, DPSCs not only differentiated into oligodendrocytes, but also promoted spinal cord regeneration by multiple neurotrophic mechanisms including the reduced apoptosis of spinal neurons and prevention of myelin degeneration (Ref. 72). Similarly, intravitreal transplantation of DPSC to a rat model of optic nerve injury resulted in a greater survival of $\mathrm{Brn} 3 \mathrm{a}$-expressing retinal ganglion cell axons compared with control (Refs 77, 79).

DPSC differentiation towards specific neuronal fates relies on chemical and environmental cues. When incubated with sonic hedgehog, FGF8 and bFGF (mimicking diffusible cues of midbrain), the cells were able to differentiate into dopaminergic neurons with up-regulated tyrosine hydroxylase, Nurrl, Engrailedl and Pitx3, and neuronal marker Map2ab (Ref. 80). DPSCs in co-culture with rat retinal explants were shown to enter into retinal neuronal fate with upregulated brain-derived neurotrophic factor (BDNF) and retinal markers (Pax6, Ascl1, PSA-NCAM and NeuroD1) (Ref. 81). Interestingly, rhodopsin, a mature photoreceptor gene, was also induced. These studies suggest that DPSC could be a lucrative source of retinal-like SCs with the capacity to differentiate into retinal neurons, and even photoreceptors (Fig. 2). This may potentially be developed into a novel strategy for rescuing retinal degeneration (associated with AMD, diabetic retinopathy, retinitis pigmentosa, macular dystrophy, artery or vein occlusion) as current treatment modalities aim at delaying the disease progress. However, once the retinal damage has commenced, retinal degeneration becomes inevitable. As such, cell replacement therapy of retinal neurons, such as rod photoreceptors, could be a potential treatment strategy. Transplantation using donor cells from photoreceptor precursors of newborn mice to precursors derived in vitro from mouse ESC and human iPSC has shown considerable retinal repair with cell integration, albeit with limitations for clinical application (Refs 82, $83,84)$.

\section{PDLSCS}

Periodontal regeneration offers clear evidence on the existence of $\mathrm{SCs}$ in dental periodontium region, which consists of gingiva, cementum of tooth root, surrounding alveolar bone and interconnecting PDL (Refs 85,86 ). PDL tissue is unique in our body as it is the only connective tissue interposed between two mineralised hard tissues (the cementum of tooth root and the alveolar socket). It surrounds the tooth root and is dynamically and continually remodelling to accommodate the growing tooth size in the alveolar bone socket during dental development. It anchors the tooth in its functional position and acts as a suspension for the tooth adapting to the mechanical load during mastication. Periodonitis is a pathological inflammation with symptoms of PDL regression leading to tooth mobility and in severe cases, tooth loss (Ref. 87). The regenerative property of PDL has been shown by the fact that only PDL, not gingival connective tissue or bone, possess cells that can derive new connection fibres between the cementum and alveolar bone (Ref. 88). It also contributes to the reformation of the complicated periodontal ligament tissue (Ref. 89). Adult PDL contains mixed cell types consisting of fibroblasts, endothelial cells, epithelial cell rests of Malassez, osteoblasts and cementoblasts as well as progenitor cells (PDLSCs) that can selfrenew and differentiate (Ref. 90).

PDLSCs are originated from the cranial neural crest and remain within PDL niche throughout adulthood. After scraping PDL tissue from the middle one-third of root surface from extracted tooth, single PDL cells were isolated by enzymatic digestion with a mixture of collagenase and trypsin. Under serum culture, the cells were plastic adherent and showed clonal proliferation as fibroblast-like cells (Ref. 49). They express markers for MSC (STRO-1, CD44, 90 and 146); ESC (OCT4, SSEA4, Nanog) and NC (nestin, Snail, Slug, p75/NTR) (Table 1) (Refs 91, 92). The percentage of SCs residing in PDL tissue is minimal when compared with other tissues. However, as PDLSCs are constantly activated for tooth/socket remodelling, they can be more proliferative once clonal growth is started. PDLSCs from young individuals may possess a greater potential to differentiate into adipocyte-like cells, rather than osteoblast-like cells. However, the effect of donor age on postnatal SC functions is not known. However, PDLSCs with retarded growth and 


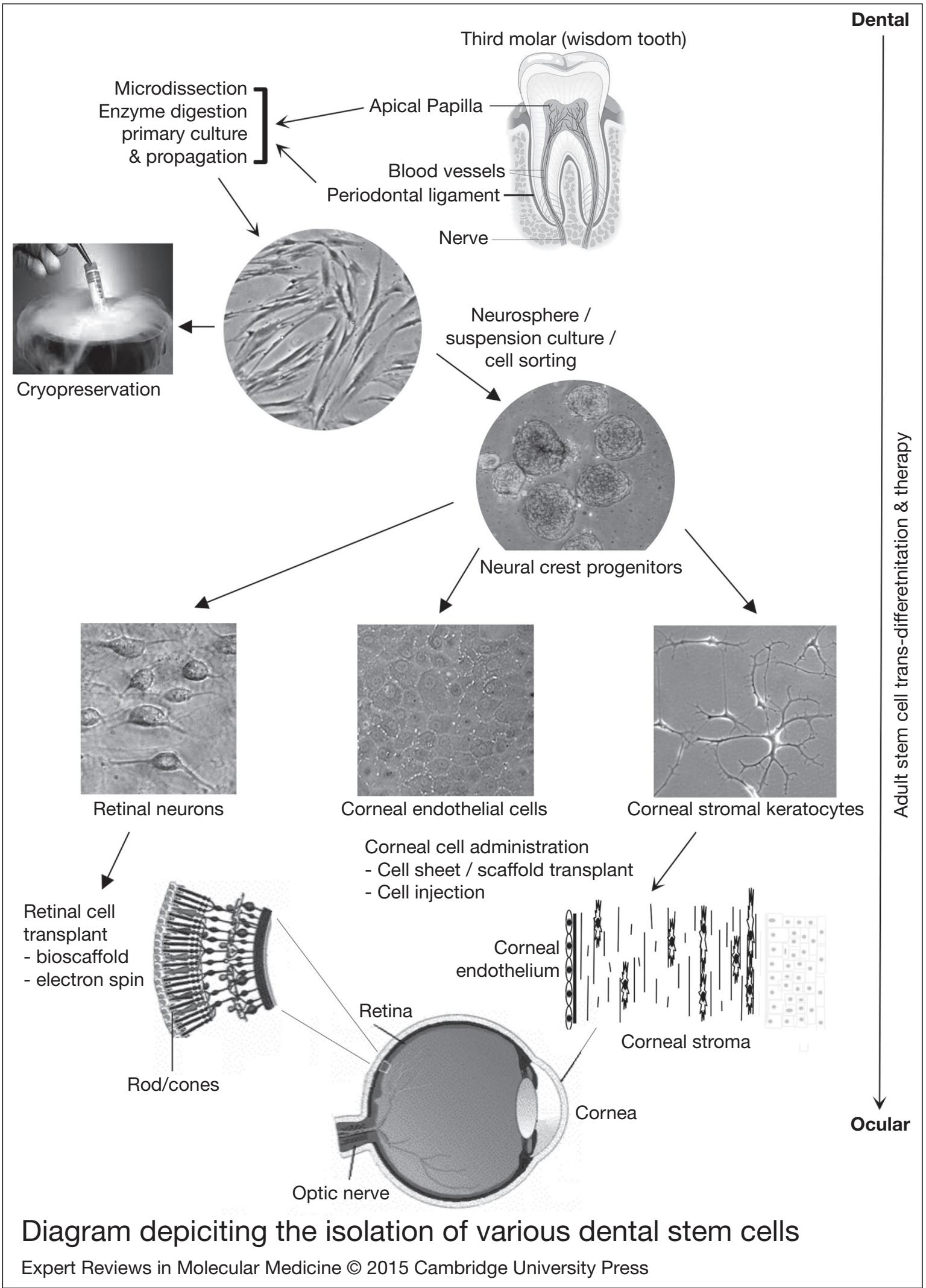

FIGURE 2.

Diagram depicting the isolation of various dental stem cells, in vitro differentiation potential to ocular cell types and its potential therapeutic uses for retinal diseases (such as age-related macular degeneration, diabetic retinopathy and Stargardt's macular dystrophy) and corneal disorders (including infectious keratitis, ectasia, corneal dystrophies and keratoconus). 
restricted differentiation ability are found in older subjects and in patients with poor oral hygiene, dentition and with a history of smoking. Tobacco smoking has been shown to chronically destroy dental SCs, in particular PDLSCs (Ref. 93). Nicotine can activate a7 nicotinic acetylcholine receptor to down-regulate Wnt pathway, resulting in reduced cell proliferation in vitro, arrested migration and differentiation capabilities (Refs 94, 95).

PDLSC exhibit multilineage differentiation potential, including adipogenic, chondrogenic, osteogenic and neurogenic lineages, when cultured under appropriate inductive conditions (Refs 49, 96, 97). In vivo transplantation of cultivated PDLSCs to immunocompromised mice formed PDL/cementum-like structure that could contribute to periodontal repair (Ref. 98). Interestingly, SSEA4-positive PDLSC contained the capacity to form cells of three embryonic germ layers: mesodermal (adipocytes, osteoblasts, chondrocytes), ectodermal (neurons) and endodermal (hepatocytes) lineages, indicating their pluripotency (Refs 99, 100). Enriched human connexin43-expressing PDLSCs were reported to express pluripotecyassociated transcription factors (Oct4, Nanog and Sox2), and markers specific for neural crest (SOX10, p75/NTR and nestin) with the capacity to generate tissue of three embryonic germ layers (Ref. 101). Recently, ABCG2-expressing PDLSC side-population has been shown to differentiate towards osteogenic, chondrogenic and adipogenic lineages (Refs 102, 103). Trans-differentiation of human PDLSC into pancreatic cell lineage was also evidenced by the formation of pancreatic islet-like clusters, insulin secretion in response to high glucose, and pancreatic marker expression (Ref. 104). Myotubular-like structures, indicative of skeletal myogenic differentiation, have been generated from human PDLSCs after 5-azacytidine induction (Ref. 105). Rat PDLSCs have also been shown to differentiate into vascular cells under phosphoinositol-3-kinase (PI3 K) pathway activation (Ref. 106). The cellular expression of c-Myc and OCT4 supports the notion of PDLSC to be a good source for iPSC generation (Ref. 107). Besides the trans-differentiation potential, PDLSC co-culture has also been shown to enhance the differentiation of dedifferentiated fat cells into osteogenic lineage (expressing RUNX2) (Ref. 108). Injection of human PDLSCs into a rat model of crushed nerve injury improved axonal regeneration and recovered sensory function, comparable with Schwann cell therapy (Ref. 109). All these findings highlight the differentiation and regenerative capacity of PDLSC.

\section{Retinal fate determination of PDLSC}

Human adult PDLSCs have the capacity to enter into retinal fate, becoming retinal progenitors exhibiting $\mathrm{Rx}^{+} \mathrm{Pax} 6^{\text {nuclear }}$ phenotype (Ref. 110). Through the formation of embryoid body-like spheroids, neural crest progenitors could be enriched and have been shown to be positive for neurogenic markers (nestin, p75/ NTR, Pax6) (Fig. 2). With the inhibition of bone morphogenic protein and Wnt signalling and under the supplementation of insulin-like growth factor (IGF) and bFGF, retinal progenitor-like cells expressing eye field transcription factors ( $\mathrm{Rx}$, PAX6, Lhx, Otx2) were generated. The associated molecular pathways were related to anterior neural plate development and mimicked the guided signalling along mammalian retinal neurogenesis. Such approach has been demonstrated using human ESC and iPSC (Refs 111, 112). Under competence modelling, the multipotent retinal progenitors can pass through a series of competent states in which different types of post-mitotic neurons are generated. Further in vitro studies showed that PDLSC-derived retinal progenitors generated mixed types of retinal neurons with a predominant photoreceptor phenotype expressing both rhodopsin and $\mathrm{Nrl}$ (Ref. 110). However, there was heterogeneity of cell type (composition and numbers within a clone), independent of the induction condition or the donor source. These variations could be genetically encoded (deterministic) or stochastically decided, and needs further clarification. Yet these exciting findings show the potential of differentiating PDLSC towards a retinal fate and could overcome the major drawbacks of the potential utilisation of ESC and iPSC.

\section{Potential to differentiate dental SCs to CSKs for the clinical management of corneal opacities}

As mentioned earlier, corneal transparency is essential for optimal vision. 'Corneal opacities', because of trauma, infection, immunological disorders and inherited diseases, is a leading cause of worldwide blindness (Refs 113, 114). Altered density and activity of CSKs and stromal nerves are common pathological features (Ref. 115). Besides the reduced light transmission, there is a compromise of stromal tissue integrity and corneal steepening leading to refractive alteration, astigmatism and increased light scattering. However, the deteriorated eyesight can be restored when the opacities are removed. Therefore, development of an alternative therapy that replaces the damaged stromal cells by healthy and functional CSKs could attain a long-term restoration of visual function (clearance of opacities for light transmittance and stromal tissue strengthening for refraction). Intrastromal CSK injection and lamellar transplantation of engineered CSKscaffold constructs are the potential routes of cell administration for the defective cornea. Minimallyinvasive intrastromal cell injection is technically much simpler than the highly-invasive corneal transplantation. Repeated injection is also feasible to improve the efficacy in cases of disease recurrence. A successful cell injection treatment requires a sufficient number of healthy CSKs $\left(\sim 10^{4}\right.$ cells per $\mu$ l per injection site experimentally) (Refs 116, 117). However, unlike corneal epithelial and endothelial cells that are expandable in culture, CSKs are difficult to propagate 
ex vivo. When isolated CSKs are cultured under serumand cytokine-supplemented condition, they proliferate but quickly transform to fibroblasts. Such change is irreversible, and the stromal fibroblasts are phenotypically and biologically different from CSKs. This is evidenced by (1) a change of cell morphology from highly dendritic shape to bipolar fibroblastic morphology and the formation of intracellular stress fibres; (2) a rapid loss of keratocyte gene expression (including keratocan, lumican, aldehyde dehydrogenases) and activation of a5-integrin, fibronectin and $\alpha$-smooth muscle actin (aSMA), indicative of fibroblast and myofibroblast transformation; and (3) a lack of production of keratan sulphate-containing proteoglycans. In addition, the actin-mediated extracellular matrix (ECM) contraction will deregulate the proper collagen fibril alignment, leading to stromal tissue opacification. The recent discovery using a mixture containing soluble amnion stromal extract, Rho kinase inhibitor and IGF1 has shown that it is possible to propagate human CSKs ex vivo without transition to fibroblasts (Ref. 118). However, the low proliferation rate remains a major limitation in its potential application for cell therapy and tissue engineering. Hence, alternative autologous cell sources with proliferative and keratocyte-differentiation capability must be sought. The identification of adult human corneal stromal SCs also offers the opportunity to develop functional keratocytes (Ref. 119). They could produce stroma-like ECM but are yet to be organized globally to produce functional stromal tissue (Refs 120, 121, 122). Lack of unique markers also makes the isolation of homogenous SC population difficult (Ref. 123). In addition, human ESC-derived neural crest-like cells have been induced to differentiate into keratocyte-like cells expressing keratocan and ALDH3A1 (Ref. 124). However, the induction efficiency and cell purity have to be optimized and the safety issue of using ESCs in clinical treatment need to be overcome. Adult bone marrow-derived and neonatal umbilical cord-derived MSC, when intrastromally injected to lumican-null $\left(\mathrm{Lum}^{-/-}\right)$and keratocan-null $\left(\mathrm{Kera}^{-/-}\right)$ mice have been shown to assume dendritic cell morphology and express CSK markers (keratocan, lumican and CD34) with an improved corneal transparency (Refs 116, 117). However, the molecular pathways associated with CSK differentiation from MSC were not delineated in these studies. More recently, SyedPicard et al. reported the capability of human DPSCs to differentiate to CSKs ex vivo under induction with bFGF, transforming growth factor $\beta 3$ (TGF $\beta 3$ ) and ascorbate-2-phosphate (Ref. 125). Intrastromal cell injection to mouse corneas demonstrated clear corneas with the production of human collagen I and keratocan. These promising data support the hypothesis that dental SCs can be a feasible autologous cell source for corneal stromal cell regeneration.

Similar to DPSC, PDLSC share the same developmental origin - cranial neural crest, as CSK. It is reasonable to consider that PDLSC can be differentiated into CSK (Fig. 2). This shortened differentiation will be advantageous over the reprogramming of adult cells to pluripotent cell stage followed by direct differentiation. This will eradicate the safety concerns over potentially uncontrolled tumour formation. In addition, PDLSC share much similarity to CSK, including (1) the expression of key stromal proteoglycans, lumican and decorin and (2) similar pro-survival responses to IGF (Refs 126, 127, 128). The success of generating CSK from PDLSC offer advantages over the ex vivo expanded CSK as PDLSC are readily propagated ex vivo and can be cryopreserved, hence offering sufficient number of viable cells for CSK differentiation for future cell therapy and stromal tissue engineering applications.

\section{Generating CECs from PDLSC}

Owing to the innate nature of human corneal endothelium, the CECs are not able to regenerate within the eye. Loss of CECs because of trauma or dystrophies of corneal endothelium, to the extent of affecting their ability to regulate corneal hydration, will result in corneal decompensation leading to blindness (Ref. 129). Partial thickness corneal endothelium transplantation (such as Descemet's stripping automated endothelial keratoplasty and Descemet membrane endothelial keratoplasty) to replace the dysfunctioned CECs can restore vision but again the outcome is restricted by the global shortage of donor corneas (Refs 38, 130). To alleviate the problem, alternative treatment approaches, such as CEC injection and tissue-engineered graft equivalents with cultivated CEC, have been proposed (Refs 130, 131, 132). However, research revolving around the use of cultivated human CEC has been slow, significantly hampered by the difficulty to propagate these primary cells efficiently (Ref. 133). Though recent reports have described relatively robust approaches in the primary CEC expansion ex vivo (Refs 134, 135), the propagation capacity is rather limited, in particular when compared with that of a SC source. There is also significant variation among donors. Several reports have shown that peripheral CECs express LGR5, a somatic SC marker, OCT4, SOX2 and PAX6, however these cells were not characterized extensively nor they were shown to possess the ability of clonogenic self-renewal (Refs 136, 137). As such, there remains an unmet need for efficient propagation of primary human CEC for potential therapeutic use.

Similar to CSK development, CEC is also derived from cranial neural crest via the intermediate periocular mesenchyme (Ref. 138). It is thus plausible to envisage the derivation of CEC from PDLSC using key developmental signalling molecules (Fig. 2). To date, although the exact mechanisms of CEC specification from neural crest or more specifically from the periocular mesenchyme have not been fully elucidated, various key 
signalling pathways and transcription factors have been identified. For example, TGF $\beta$ signalling is associated with the formation of corneal endothelium (Ref. 139). Along with RA signalling, the induction of key transcription factors (PITX2 and FOXC1) is necessary for CEC development (Refs 140, 141). Recent transcriptome profiling comparing human CEC and CSK further suggested the involvement of Wnt signalling (Refs 142, 143). However, deeper understanding of these pathways within the context of CEC development and studies into the temporal involvement of various signalling molecules are required in understanding the complex differentiation of PDLSC towards CEC.

\section{Tooth banking: the first step to future tissue engineering}

The harvesting of dental SCs (from extracted teeth) is a significant upside when compared with bone marrow, umbilical cord or adipose-derived MSCs, as well as other forms of adult SCs that require more invasive procedures, which are usually associated with pain and the risk of adverse events. Although dental SCs can be found in all teeth, the third molar is the most common source for cell isolation, as it is the last tooth to develop, and is normally in a premature stage of development. In most humans, it is usually unused hence the SC quality should be well preserved. The relatively large tooth volume and surface area generally yield more dental pulp tissue for the isolation of DPSC and PDL for PDLSC. Although the percentage of adult SCs usually decreases with age, dental SC population is always present, even in older subjects (Ref. 61). Wisdom tooth extraction is generally performed worldwide and these extracted teeth are merely disposed as medical waste. Hence, their use for SC research and application has minimal ethical issues. With such advantage, the concept of 'tooth banking' was initiated in 1966 (Ref. 144). There have been several reports on tooth cryopreservation but very limited data have been provided for subsequent isolation efficiency of dental SCs. Lee et al. showed that DPSCs were viable when isolated from cryopreserved teeth under controlled cooling condition without cryoprotectants (Ref. 65). The cooling procedure was performed using a programmable freezer coupled to a magnetic field generated by $75 \mathrm{~mA}$ electric current and the temperature was controlled at $-5^{\circ} \mathrm{C}$ for $15 \mathrm{~min}$, followed by temperature drop at $0.5^{\circ} \mathrm{C} / \mathrm{min}$ to $-30^{\circ} \mathrm{C}$ and the final storage temperature at $-150^{\circ} \mathrm{C}$. Hence, the absence of detailed preservation methodology for teeth and/or dental SCs remains a significant limitation. Further research on this aspect will allow the design of proper storage of autologous SCs for future use, guaranteeing donor matching without immunological or genetic incompatibility. Besides a facility development to obtain high-quality dental SCs, the concept of tooth saving and banking should begin with the dentist and dental caregiver as this could impact their practice protocol. They are in a pivotal position to control the quality of tooth materials, which is affected by a number of daily habits. General public should be provided with information regarding the option of tooth or dental SC banking, emerging research outcome and potential clinical use. They need to be educated with ways to maintain oral hygiene and eliminate the contaminating oral flora. Smoking is a great impact to the healthy dental SC population and restricts their differentiation potential (Ref. 95). Hence, eligible patients should be educated to minimise smoking, which can preserve the health of SC population and this would be beneficial to the patients' future.

\section{Conclusion}

Dental SCs could be a milestone in personalised regenerative medicine. Advances in the isolation and understanding of these SC populations as well as their differentiation capabilities could open up new fields of research and novel treatment modalities for degenerative and aging disorders in ophthalmology and other clinical areas.

\section{Financial support}

This work was supported by Biomedical Research Council Translational Clinical Research Partnership Grant - TCR0101673 and NMRC/TCR/1020-SERI/ 2013, and SingHealth Foundation Transitional Grant SHF/FG585P/2014.

\section{Conflict of Interest}

None.

\section{References}

1. Shackleton M. et al. (2006) Generation of a functional mammary gland from a single stem cell. Nature 439, 84-88

2. Leong K.G. et al. (2008) Generation of a prostate from a single adult stem cell. Nature 456, 804-808

3. Liu Y. et al. (2013) Generation of functional organs from stem cells. Cell Regeneration (London) 2, 1

4. Takebe T. et al. (2014) Generation of a vascularized and functional human liver from an iPSC-derived organ bud transplant. Nature Protocols 9, 396-409

5. Gnecchi M. et al. (2008) Paracrine mechanisms in adult stem cell signaling and therapy. Circulation Research 103, 12041219

6. Levi B. et al. (2011) Human adipose-derived stromal cells stimulate autogenous skeletal repair via paracrine Hedgehog signaling with calvarial osteoblasts. Stem Cells and Development 20, 243-257

7. Lebkowski J. (2011) GRNOPC1: the world's first embryonic stem cell-derived therapy. Interview with Jane Lebkowski. Regenerative Medicine 6(6 Suppl), 11-13

8. Watson R.A., Yeung T.M. (2011) What is the potential of oligodendrocyte progenitor cells to successfully treat human spinal cord injury? BMC Neurology 11, 113

9. Schwartz S.D. et al. (2015) Human embryonic stem cellderived retinal pigment epithelium in patients with age-related macular degeneration and Stargardt's macular dystrophy: follow-up of two open-label phase 1/2 studies. Lancet 385, 509-516

10. Yamanaka S. (2012) Induced pluripotent stem cells: past, present, and future. Cell Stem Cell 10, 678-684

11. Rao M.S., Malik N. (2012) Assessing iPSC reprogramming methods for their suitability in translational medicine. Journal of Cellular Biochemistry 113, 3061-3068 
12. Walia B. et al. (2012) Induced pluripotent stem cells: fundamentals and applications of the reprogramming process and its ramifications on regenerative medicine. Stem Cell Reviews 8, 100-115

13. Shtrichman R., Germanguz I., Itskovitz-Eldor J. (2013) Induced pluripotent stem cells (iPSCs) derived from different cell sources and their potential for regenerative and personalized medicine. Current Molecular Medicine 13, 792-805

14. Marchetto M.C. et al. (2009) Transcriptional signature and memory retention of human-induced pluripotent stem cells. PLOS ONE 4, e7076

15. Rouhani F. et al. (2014) Genetic background drives transcriptional variation in human induced pluripotent stem cells. PLoS Genetics 10, e1004432

16. Rohani L. et al. (2014) The aging signature: a hallmark of induced pluripotent stem cells? Aging Cell 13, 2-7

17. Barker N., Bartfeld S., Clevers H. (2010) Tissue-resident adult stem cell populations of rapidly self-renewing organs. Cell Stem Cell 7, 656-670

18. Barrilleaux B. et al. (2006) Review: ex vivo engineering of living tissues with adult stem cells. Tissue Engineering 12, 3007-3019

19. Majhail N.S., Rizzo J.D. (2013) Surviving the cure: long term followup of hematopoietic cell transplant recipients. Bone Marrow Transplant 48, 1145-1151

20. Huang H., Chen L., Sanberg P. (2010) Cell therapy from bench to bedside translation in CNS neurorestoratology era. Cell Medicine 1, 15-46

21. Glenn J.D., Whartenby K.A. (2014) Mesenchymal stem cells: emerging mechanisms of immunomodulation and therapy. World Journal of Stem Cells 6, 526-539

22. Wei X. et al. (2013) Mesenchymal stem cells: a new trend for cell therapy. Acta Pharmacologica Sinica 34, 747-754

23. Hayashi T. et al. (2013) Autologous mesenchymal stem cellderived dopaminergic neurons function in parkinsonian macaques. Journal of Clinical Investigation 123, 272-284

24. Giordano R. et al. (2014) Autologous mesenchymal stem cell therapy for progressive supranuclear palsy: translation into a phase I controlled, randomized clinical study. Journal of Translational Medicine 12, 14

25. Bouwens L., Houbracken I., Mfopou J.K. (2013) The use of stem cells for pancreatic regeneration in diabetes mellitus. Nature Reviews Endocrinology 9, 598-606

26. Soria B. et al. (2000) Insulin-secreting cells derived from embryonic stem cells normalize glycemia in streptozotocininduced diabetic mice. Diabetes 49, 157-162

27. Lumelsky N. et al. (2001) Differentiation of embryonic stem cells to insulin-secreting structures similar to pancreatic islets. Science 292, 1389-1394

28. Jeon K. et al. (2012) Differentiation and transplantation of functional pancreatic beta cells generated from induced pluripotent stem cells derived from a type 1 diabetes mouse model. Stem Cells and Development 21, 2642-2655

29. Wei R. et al. (2013) Insulin-producing cells derived from human embryonic stem cells: comparison of definitive endoderm- and nestin-positive progenitor-based differentiation strategies. PLoS ONE 8, e72513

30. Choi K.S. et al. (2005) In vitro trans-differentiation of rat mesenchymal cells into insulin-producing cells by rat pancreatic extract. Biochemical and Biophysical Research Communications 330, 1299-1305

31. Oh S.H. et al. (2004) Adult bone marrow-derived cells transdifferentiating into insulin-producing cells for the treatment of type I diabetes. Laboratory Investigation 8, 607-617

32. Kamdar F. et al. (2012) Cellular therapy promotes endogenous stem cell repair. Canadian Journal of Physiology and Pharmacology 90, 1335-1344

33. Mariotti S.P. (2012) Global Data on Visual Impairment 2010. World Health Organization

34. Oliva M.S., Schottman T., Gulati M. (2012) Turning the tide of corneal blindness. Indian Journal of Ophthalmology 60, 423-427

35. Pascolini D., Mariotti S.P. (2012) Global estimates of visual impairment: 2010. British Journal of Ophthalmology 96, 614-618

36. Anshu A. et al. (2009) Outcomes of therapeutic deep lamellar keratoplasty and penetrating keratoplasty for advanced infectious keratitis: a comparative study. Ophthalmology 116, 615-623

37. Ang M. et al. (2012) Indications, outcomes, and risk factors for failure in tectonic keratoplasty. Ophthalmology 119, 1311-1319

38. Tan D.T. et al. (2012) Corneal transplantation. Lancet 379, 1749-1761

39. Yuen L.H. et al. (2011) Hemi-automated lamellar keratoplasty (HALK). British Journal of Ophthalmology 95, 1513-1518

40. Zhou Q. et al. (2008) In vivo reprogramming of adult pancreatic exocrine cells to beta-cells. Nature 455, 627-632

41. Cavelti-Weder C. et al. (2014) Direct lineage conversion of pancreatic exocrine to endocrine Beta cells in vivo with defined factors. Methods in Molecular Biology 1150, 247-262

42. Ieda M. et al. (2010) Direct reprogramming of fibroblasts into functional cardiomyocytes by defined factors. Cell 142, 375-386

43. Torper O. et al. (2013) Generation of induced neurons via direct conversion in vivo. Proceedings of the National Academy of Sciences of the United States of America 110, 7038-7043

44. Galli D., Vitale M., Vaccarezza M. (2014) Bone marrowderived mesenchymal cell differentiation toward myogenic lineages: facts and perspectives. Biomed Research International 2014, 762695

45. Harada H. et al. (1999) Localization of putative stem cells in dental epithelium and their association with Notch and FGF signaling. Journal of Cell Biology 147, 105-120

46. Chavez M.G. et al. (2014) Isolation and culture of dental epithelial stem cells from the adult mouse incisor. Journal of Visualized Experiments 87, doi:10.3791/51266

47. Gronthos S. et al. (2000) Postnatal human dental pulp stem cells (DPSCs) in vitro and in vivo. Proceedings of the National Academy of Sciences of the United States of America 97, 13625-13630

48. Morsczeck C. et al. (2005) Isolation of precursor cells (PCs) from human dental follicle of wisdom teeth. Matrix Biology 24, 155-165

49. Seo B.M. et al. (2004) Investigation of multipotent postnatal stem cells from human periodontal ligament. Lancet 364, 149-155

50. Huang G.T. et al. (2008) The hidden treasure in apical papilla: the potential role in pulp/dentin regeneration and bioroot engineering. Journal of Endodontics 34, 645-651

51. Miura M. et al. (2003) SHED: stem cells from human exfoliated deciduous teeth. Proceedings of the National Academy of Sciences of the United States of America 100, 5807-5812

52. Huang G.T., Gronthos S., Shi S. (2009) Mesenchymal stem cells derived from dental tissues vs. those from other sources: their biology and role in regenerative medicine. Journal of Dental Research 88, 792-806

53. Laberge T., Cheung H.S. (2011) Multipotent dental stem cells: an alternative adult derived stem cell source for regenerative medicine. In Embryonic Stem Cells - Differentiation and Pluripotent Alternatives (Kallas M.S. ed.), ISBN: 978953-307-632-4, InTech, doi: 10.5772/24918. Available from: http://www.intechopen.com/books/embryonic-stem-cells-differ entiation-and-pluripotentalternatives/multipotent-dental-stemcells-an-alternative-adult-derived-stem-cell-source-for-regener ativemedicine

54. Ibarretxe G. et al. (2012) Neural crest stem cells from dental tissues: a new hope for dental and neural regeneration. Stem Cells International 2012, 103503

55. Volponi A.A., Sharpe P.T. (2013) The tooth - a treasure chest of stem cells. British Dental Journal 215, 353-358

56. Creuzet S., Vincent C., Couly G. (2005) Neural crest derivatives in ocular and periocular structures. International Journal of Developmental Biology 49, 161-171

57. Gage P.J. et al. (2005) Fate maps of neural crest and mesoderm in the mammalian eye. Investigative Ophthalmology \& Visual Science 46, 4200-4208

58. Chao J.R., Bronner M.E., Lwigale P.Y. (2013) Human fetal keratocytes have multipotent characteristics in developing avian embryo. Stem Cells and Development 22, 2186-2195

59. Gronthos S. et al. (2011) A method to isolate and culture expand human dental pulp stem cells. Methods in Molecular Biology 698, 107-121 
60. Tatullo M. et al. (2014) Dental pulp stem cells: function, isolation and applications in regenerative medicine. Journal of Tissue Engineering and Regenerative Medicine. Published online in Wiley Online Library (wileyonlinelibrary.com). doi: 10.1002/term.1899

61. Atari M. et al. (2012) Dental pulp of the third molar: a new source of pluripotent-like stem cells. Journal of Cell Science 125, 3343-3356

62. Hilkens P. et al. (2013) Effect of isolation methodology on stem cell properties and multilineage differentiation potential of human dental pulp stem cells. Cell and Tissue Research 353, 65-78

63. Lizier N.F. et al. (2012) Scaling-up of dental pulp stem cells isolated from multiple niches. PLOS ONE 7, e39885

64. Woods E.J. et al. (2009) Optimized cryopreservation method for human dental pulp-derived stem cells and their tissues of origin for banking and clinical use. Cryobiology 59, 150-157

65. Lee S.Y. et al. (2010) Effects of cryopreservation of intact teeth on the isolated dental pulp stem cells. Journal of Endodontics 36, 1336-1340

66. d'Aquino R. et al. (2007) Human postnatal dental pulp cells co-differentiate into osteoblasts and endotheliocytes: a pivotal synergy leading to adult bone tissue formation. Cell Death \& Differentiation 14, 1162-1171

67. Mori G. et al. (2011) Dental pulp stem cells: osteogenic differentiation and gene expression. Annals of the New York Academy of Sciences 1237, 47-52

68. Nozaki T., Ohura K. (2011) Gene expression profile of dental pulp cells during differentiation into an adipocyte lineage. Journal of Pharmacological Sciences 115, 354-363

69. Atari M. et al. (2012) The enhancement of osteogenesis through the use of dental pulp pluripotent stem cells in 3D. Bone 50, 930-941

70. Monteiro B.G. et al. (2009) Human immature dental pulp stem cells share key characteristic features with limbal stem cells. Cell Proliferation 42, 587-594

71. Gomes J.A. et al. (2010) Corneal reconstruction with tissueengineered cell sheets composed of human immature dental pulp stem cells. Investigative Ophthalmology \& Visual Science 51, 1408-1414

72. Sakai K. et al. (2012) Human dental pulp-derived stem cells promote locomotor recovery after complete transection of the rat spinal cord by multiple neuro-regenerative mechanisms. Journal of Clinical Investigation 122, 80-90

73. Young F., Sloan A., Song B. (2013) Dental pulp stem cells and their potential roles in central nervous system regeneration and repair. Journal of Neuroscience Research 91, 1383-1393

74. Zainal Ariffin S.H. et al. (2013) Differentiation of dental pulp stem cells into neuron-like cells in serum-free medium. Stem Cells International 2013, 250740

75. Arthur A. et al. (2008) Adult human dental pulp stem cells differentiate toward functionally active neurons under appropriate environmental cues. Stem Cells 26, 1787-1795

76. Kiraly M. et al. (2009) Simultaneous PKC and cAMP activation induces differentiation of human dental pulp stem cells into functionally active neurons. Neurochemistry International 55, 323-332

77. Mead B. et al. (2013) Intravitreally transplanted dental pulp stem cells promote neuroprotection and axon regeneration of retinal ganglion cells after optic nerve injury. Investigative Ophthalmology \& Visual Science 54, 7544-7556

78. Mead B. et al. (2014) Paracrine-mediated neuroprotection and neuritogenesis of axotomised retinal ganglion cells by human dental pulp stem cells: comparison with human bone marrow and adipose-derived mesenchymal stem cells. PLOS ONE $\mathbf{9}$, e109305

79. Mead B. et al. (2014) Dental pulp stem cells, a paracrinemediated therapy for the retina. Neural Regeneration Research 9, 577-578

80. Kanafi M. et al. (2014) Midbrain cues dictate differentiation of human dental pulp stem cells towards functional dopaminergic neurons. Journal of Cellular Physiology 229, 1369-1377

81. Bray A.F. et al. (2014) Human dental pulp stem cells respond to cues from the rat retina and differentiate to express the retinal neuronal marker rhodopsin. Neuroscience 280, 142-155

82. Lamba D.A., Gust J., Reh T.A. (2009) Transplantation of human embryonic stem cell-derived photoreceptors restores some visual function in Crx-deficient mice. Cell Stem Cell 4, 73-79

83. Pearson R.A. et al. (2012) Restoration of vision after transplantation of photoreceptors. Nature 485, 99-103

84. Gonzalez-Cordero A. et al. (2013) Photoreceptor precursors derived from three-dimensional embryonic stem cell cultures integrate and mature within adult degenerate retina. Nature Biotechnology 31, 741-747

85. Maeda H. et al. (2011) Promise of periodontal ligament stem cells in regeneration of periodontium. Stem Cell Research \& Therapy 2, 33

86. Han J. et al. (2014) Assessment of the regenerative potential of allogeneic periodontal ligament stem cells in a rodent periodontal defect model. Journal of Periodontal Research 49, 333-345

87. Darveau R.P. (2010) Periodontitis: a polymicrobial disruption of host homeostasis. Nature Reviews Microbiology 8, 481-490

88. Trombelli L. (1999) Periodontal regeneration in gingival recession defects. Periodontology 2000 19, 138-150

89. Boyko G.A., Melcher A.H., Brunette D.M. (1981) Formation of new periodontal ligament by periodontal ligament cells implanted in vivo after culture in vitro. A preliminary study of transplanted roots in the dog. Journal of Periodontal Research 16, 73-88

90. Bright R. et al. (2015) Periodontal ligament-derived cells for periodontal regeneration in animal models: a systematic review. Journal of Periodontal Research 50, 160-172

91. Trubiani O. et al. (2010) Expression profile of the embryonic markers nanog, OCT-4, SSEA-1, SSEA-4, and frizzled-9 receptor in human periodontal ligament mesenchymal stem cells. Journal of Cell Physiology 225, 123-131

92. Mrozik K. et al. (2010) A method to isolate, purify, and characterize human periodontal ligament stem cells. Methods in Molecular Biology 666, 269-284

93. Bergstrom J. (2004) Tobacco smoking and chronic destructive periodontal disease. Odontology 92, 1-8

94. Zhou Z. et al. (2013) Nicotine deteriorates the osteogenic differentiation of periodontal ligament stem cells through alpha7 nicotinic acetylcholine receptor regulating Wnt pathway. PLoS ONE 8, e83102

95. Ng T.K. et al. (2015) Cigarette smoking hinders human periodontal ligament-derived stem cell proliferation, migration and differentiation potentials. Scientific Reports 5, 7828

96. Sonoyama W. et al. (2006) Mesenchymal stem cell-mediated functional tooth regeneration in swine. PLOS ONE 1, e79

97. Tomokiyo A et al. (2012) A multipotent clonal human periodontal ligament cell line with neural crest cell phenotypes promotes neurocytic differentiation, migration, and survival. Journal of Cellular Physiology 227, 2040-2050

98. Lei M. et al. (2014) Mesenchymal stem cell characteristics of dental pulp and periodontal ligament stem cells after in vivo transplantation. Biomaterials 35, 6332-6343

99. Kawanabe N. et al. (2010) Isolation of multipotent stem cells in human periodontal ligament using stage-specific embryonic antigen-4. Differentiation 79, 74-83

100. Fukushima H. et al. (2012) SSEA-4 is a marker of human deciduous periodontal ligament stem cells. Journal of Dental Research 91, 955-960

101. Pelaez D., Huang C.Y., Cheung H.S. (2013) Isolation of pluripotent neural crest-derived stem cells from adult human tissues by connexin-43 enrichment. Stem Cells Development 22, 2906-2914

102. Zhang J. et al. (2012) The effect of aging on the pluripotential capacity and regenerative potential of human periodontal ligament stem cells. Biomaterials 33, 6974-6986

103. Ninomiya T. et al. (2014) Enhanced bone-forming activity of side population cells in the periodontal ligament. Cell Transplantation 23, 691-701

104. Lee J.S. et al. (2014) Transdifferentiation of human periodontal ligament stem cells into pancreatic cell lineage. Cell Biochemistry and Function 32, 605-611

105. Song M. et al. (2012) Skeletal myogenic differentiation of human periodontal ligament stromal cells isolated from orthodontically extracted premolars. Korean Journal of Orthodontics 42, 249-254

106. Okubo N. et al. (2010) Vascular cell-like potential of undifferentiated ligament fibroblasts to construct vascular cellspecific marker-positive blood vessel structures in a PI3 K 
activation-dependent manner. Journal of Vascular Research 47, 369-383

107. Nomura Y. et al. (2012) Human periodontal ligament fibroblasts are the optimal cell source for induced pluripotent stem cells. Histochemistry and Cell Biology 137, 719-732

108. Tansriratanawong K. et al. (2014) Co-culture with periodontal ligament stem cells enhances osteogenic gene expression in de-differentiated fat cells. Human Cell 27, 151-161

109. Li B. et al. (2013) Human periodontal ligament stem cells repair mental nerve injury. Neural Regeneration Research $\mathbf{8}$, 2827-2837

110. Huang L. et al. (2013) Directing adult human periodontal ligament-derived stem cells to retinal fate. Investigative Ophthalmology \& Visual Science 54, 3965-3974

111. Lamba D.A. et al. (2006) Efficient generation of retinal progenitor cells from human embryonic stem cells. Proceedings of the National Academy of Sciences of the United States of America 103, 12769-12774

112. Lamba D.A et al. (2010) Generation, purification and transplantation of photoreceptors derived from human induced pluripotent stem cells. PLoS ONE 5, e8763

113. Rezende R.A. et al. (2004) Congenital corneal opacities in a cornea referral practice. Cornea 23, 565-570

114. Michaeli A., Markovich A., Rootman D.S. (2005) Corneal transplants for the treatment of congenital corneal opacities. Journal of Pediatric Ophthalmology and Strabismus 42, 34-44

115. Meek K.M. et al. (2003) Transparency, swelling and scarring in the corneal stroma. Eye (London) 17, 927-936

116. Liu H. et al. (2010) Cell therapy of congenital corneal diseases with umbilical mesenchymal stem cells: lumican null mice. PLoS ONE 5, e10707

117. Liu H. et al. (2012) Bone marrow mesenchymal stem cells can differentiate and assume corneal keratocyte phenotype. Journal of Cellular and Molecular Medicine 16, 11141124

118. Yam G.H. et al. (2015) Ex Vivo Propagation of human corneal stromal "activated keratocytes" for tissue engineering. Cell Transplantation 24, 1845-1861

119. Pinnamaneni N., Funderburgh J.L. (2012) Concise review: stem cells in the corneal stroma. Stem Cells 30, 1059-1063

120. Du Y. et al. (2009) Stem cell therapy restores transparency to defective murine corneas. Stem Cells 27, 1635-1642

121. Wu J. et al. (2012) The engineering of organized human corneal tissue through the spatial guidance of corneal stromal stem cells. Biomaterials 33, 1343-1352

122. Wu J. et al. (2014) Corneal stromal stem cells versus corneal fibroblasts in generating structurally appropriate corneal stromal tissue. Experimental Eye Research 120, 71-81

123. Hashmani K. et al. (2013) Characterization of corneal stroma stem cells with the potential for epithelial transdifferentiation. Stem Cell Research \& Therapy 4, 75

124. Chan A.A. et al. (2013) Differentiation of human embryonic stem cells into cells with corneal keratocyte phenotype. PLOS ONE 8, e56831

125. Syed-Picard F.N. et al. (2015) Dental pulp stem cells: a new cellular resource for corneal stromal regeneration. Stem Cells Translational Medicine 4, 276-285

126. Han X., Amar S. (2003) IGF-1 signaling enhances cell survival in periodontal ligament fibroblasts vs. gingival fibroblasts. Journal of Dental Research 82, 454-459

127. Matheson S., Larjava H., Hakkinen L. (2005) Distinctive localization and function for lumican, fibromodulin and decorin to regulate collagen fibril organization in periodontal tissues. Journal of Periodontal Research 40, 312-324
128. Etheredge L., Kane B.P., Hassell J.R. (2009) The effect of growth factor signaling on keratocytes in vitro and its relationship to the phases of stromal wound repair. Investigative Ophthalmology \& Visual Science 50, 3128-3136

129. Peh G.S. et al. (2011) Human corneal endothelial cell expansion for corneal endothelium transplantation: an overview. Transplantation 91, 811-819

130. Proulx S. et al. (2009) Transplantation of a tissue-engineered corneal endothelium reconstructed on a devitalized carrier in the feline model. Investigative Ophthalmology \& Visual Science 50, 2686-2694

131. Koizumi N., Okumura N., Kinoshita S. (2012) Development of new therapeutic modalities for corneal endothelial disease focused on the proliferation of corneal endothelial cells using animal models. Experimental Eye Research 95, 60-67

132. Levis H.J. et al. (2012) Plastic compressed collagen as a novel carrier for expanded human corneal endothelial cells for transplantation. PLOS ONE 7, e50993

133. Peh G.S. et al. (2011) Cultivation of human corneal endothelial cells isolated from paired donor corneas. PLOS ONE 6, e 28310

134. Nakahara M. et al. (2013) Corneal endothelial expansion promoted by human bone marrow mesenchymal stem cellderived conditioned medium. PLoS ONE 8, e69009

135. Peh G.S. et al. (2015) Propagation of human corneal endothelial cells: a novel dual media approach. Cell Transplantation 24, 287-304

136. Barker N. et al. (2007) Identification of stem cells in small intestine and colon by marker gene Lgr5. Nature 449, 1003-1007

137. McGowan S.L. et al. (2007) Stem cell markers in human posterior limbus and corneal endothelium of unwounded and wounded corneas. Molecular Vision 13, 1984-2000

138. Kao W.W., Liu H., Zhang J. (2013) Wakayama symposium: challenges of future research in ocular surface cell biology. The Ocular Surface 11, 19-24

139. Ittner L.M. et al. (2005) Compound developmental eye disorders following inactivation of TGFbeta signaling in neuralcrest stem cells. Journal of Biology 4, 11

140. Matt N. et al. (2005) Retinoic acid-dependent eye morphogenesis is orchestrated by neural crest cells. Development 132, 4789-4800

141. Matt N. et al. (2008) Impairing retinoic acid signalling in the neural crest cells is sufficient to alter entire eye morphogenesis. Developmental Biology 320, 140-148

142. Chen Y. et al. (2013) Identification of novel molecular markers through transcriptomic analysis in human fetal and adult corneal endothelial cells. Human Molecular Genetics 22, 1271-1279

143. Chng Z. et al. (2013) High throughput gene expression analysis identifies reliable expression markers of human corneal endothelial cells. PLOS ONE 8, e67546

144. Coburn R.J., Henriques B.L., Francis L.E. (1966) The development of an experimental tooth bank using deep freeze and tissue culture techniques. Journal of Oral Therapeutics and Pharmacology 2, 445-450

*Corresponding author:

Associate Professor Jodhbir S. Mehta,

Singapore National Eye Centre,

11 Third Hospital Avenue,

Singapore 168751, Singapore.

Tel: +65 62277255 ;

Fax: + 6562277290

E-mail: jodmehta@gmail.com 